\title{
Prospective Study of Radiological and Functional Outcome of Closed Subtrochanteric Fracture Fixation with Proximal Humerus Interlocking Plate in Adolescent Patients
}

By Amit Bansal

Abstract- Background: Subtrochanteric fractures constitute $1 \%$ in children. Subtrochanteric fractures in pediatric age defined as $10 \%$ length of total femur below the lesser trochanter. Mostly these fractures are unstable types. There are various treatment options available for the management of this fracture, depending on the age group of the patient. But there is no welldefined management for adolescent subtrochanteric fractures.

Methods: This study includes five patients present to orthopedic emergency with closed subtrochanteric fracture without distal neurological deficit. Patients were managed operatively after informed consent with proximal humerus locking plate under regional anesthesia. Postoperatively patients were kept non weight bearing with in-bed exercises. Follow-up was done at 2, 6, 12, 24, and 36 weeks. Patients were evaluated as functional and radiologically.

Keywords: subtrochanteric fracture, proximal humerus locking plate, adolescent patients.

GJMR-H Classification: NLMC Code: WE 168

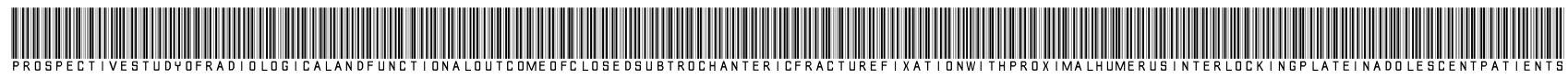

Strictly as per the compliance and regulations of:

(C) 2020. Amit Bansal. This is a research/review paper, distributed under the terms of the Creative Commons AttributionNoncommercial 3.0 Unported License http://creativecommons.org/licenses/by-nc/3.0/), permitting all non-commercial use, distribution, and reproduction in any medium, provided the original work is properly cited. 


\title{
Prospective Study of Radiological and Functional Outcome of Closed Subtrochanteric Fracture Fixation with Proximal Humerus Interlocking Plate in Adolescent Patients
}

\author{
Amit Bansal
}

\begin{abstract}
Background: Subtrochanteric fractures constitute $1 \%$ in children. Subtrochanteric fractures in pediatric age defined as $10 \%$ length of total femur below the lesser trochanter. Mostly these fractures are unstable types. There are various treatment options available for the management of this fracture, depending on the age group of the patient. But there is no well-defined management for adolescent subtrochanteric fractures.
\end{abstract}

Methods: This study includes five patients present to orthopedic emergency with closed subtrochanteric fracture without distal neurological deficit. Patients were managed operatively after informed consent with proximal humerus locking plate under regional anesthesia. Postoperatively patients were kept non weight bearing with in-bed exercises. Follow-up was done at 2, 6, 12, 24, and 36 weeks. Patients were evaluated as functional and radiologically.

Results: There were five patients included in this study. There was no gender difference in the incidence of fracture. The average time of union was 11.4 wks in the adolescent age group. There was no other early and late complication. Ambulation was done with protected weight-bearing with some support at an average of 10 wks. Follow-up was done until nine months. The final average harris hip score was 91 .

Conclusion: Proximal humerus locking plate found to be an excellent choice of implant for any pattern of subtrochanteric fracture. The plate surface found to be well contoured according to the proximal femur lateral surface.

Keywords: subtrochanteric fracture, proximal humerus locking plate, adolescent patients.

\section{INTRODUCTION}

P ediatric subtrochanteric fracture defined as 10\% percent the length of the whole femur below the lesser trochanter. ${ }^{1}$ Subtrochanteric fractures constitute $1 \%$ in children. ${ }^{1,2}$ Adolescent subtrochanteric fractures are unusual and have received less attention in literature. ${ }^{1-3}$ There are various deforming forces around this fracture like proximal fracture tends to flex, abduct, and external rotate and distal fragment adducts. ${ }^{1}$ Due to various deforming forces around this fracture, this fracture requires special attention. There are number of management available for this fracture in each age group. Infants are with Pavlik harness, children (6month

Author: MBBS, DNB (Orthopaedics), Senior Resident, Department of Orthopaedics, VMMC \& Safdarjung Hospital, New Delhi, India.

e-mail: amitb06007@gmail.com
-5 years) with a hip spica cast. ${ }^{1}$ The dilemma starts after the age of 10 years. ${ }^{1}$ There is no definite consensus available for this age group. Management of this fracture in the adolescent age group is deficient. Traction alone found unsatisfactory and incapable of providing reduction and stability. According to literature fixation with elastic nailing is inadequate and had various complication like malunion and shortening. ${ }^{1,4,5}$

\section{Aims And OBjectives}

To study the functional and radiological outcomes of open reduction and internal fixation of closed subtrochanteric fracture with a proximal humerus locking plate.

\section{ili. Materials and Methods}

\section{a) Study Area}

The study was done from November/2016 to June/2018 at Safdarjung Hospital New Delhi.

\section{b) Study Population}

In our series age of patient was 10 to 20 years with the diagnosis of closed subtrochanteric fracture attending the Department of Orthopaedics, Safdarjung Hospital New Delhi managed surgically.

\section{c) Sample Size and Sample Technique}

Five adolescent patients attended the hospitals from November/2016 to June/2018 presented in emergency with the closed subtrochanteric fracture.

\section{d) Data Collection Technique and Tools}

Five adolescent patients operated with open reduction and internal fixation with a proximal humerus locking plate, followed by physiotherapy and range of movement exercises. Follow-up was done at $2 \mathrm{wks}$, $6 w k s, 12 w k s, 24 w k s$, and 36 wks. All patients gave their informed consent.

\section{Inclusion Criteria}

1. Age $10-20 \mathrm{yrs}$,

2. Closed fracture

3. Fracture without a distal neurovascular deficit. 


\section{Exclusion Criteria}

1. Patient with other life-threatening comorbidities

2. Previous hip surgery

3. Pathological fracture

4. Previous hip pathology.

Preoperative: Each patient had given the informed consent. We had sent blood investigation for preanesthetic clearence. Temporary Bohlerbraun splint with skin traction applied to relieve some pain and improve some fracture deformity.

Perioperative: Implant choice -Under general anesthesia, open reduction and internal fixation performed with proximal humerus interlocking osteosynthesis plate through lateral approach to the thigh. Proximal humerus locking plate found to have a low profile and narrow which is a right amount of thickness for adolescent patients.

Procedure: 1. Under general anesthesia, patient positioned in a lateral decubitus position. The patient's affected limb painted and draped.

Dead lateral incision over thigh given. Good hemostasis achieved. Lift Vastus lateral is from linea aspra instead of splitting it. Partial proximal origin of vastus lateral is removed to make space for the plate. Open reduction was done with temporary $\mathrm{k}$ wire fixation. Proximal locking screws were kept short of femoral head physis to avoid its injury.

Fracture was fixed with Proximal humerus interlocking plate. A thorough wash was given. An incision was closed in layers with sunction drain insitu.

Postoperative: First dressing was done after 48 hrs of surgery. Immediate postoperative $\mathrm{x}$ rays were taken. Static quadriceps exercise, knee range of motion exercises, and ankle range of motion exercises were started after $24 \mathrm{hrs}$ from time of surgery. Patients were discharged on the fourth postoperative day.

Follow up: Patients were advised for strict non-weight bearing and in bed ambulation exercises. Patients were followed at 2wks, 6 wks, 12 wks, and 24 wks as outpatients.

2 wks - Sutures were removed at 2 wk follow up x-rays. In bed ambulatory exercises were continued.

6 wks- Follow up $x$ rays were done to assess radiologically. Harris hip scoring was done. As the patient was adolescent, walker assisted walking was started late.

12 wks- follow up $\mathrm{x}$ rays and Harris hip scoring was done. Non-weight bearing walking with walker support was started.

24 wks- As patients were adolescent, Partial weight bearing was started after achieving radiological and functional improvement at 24 wks.

36 wks - Radiological and functional evaluation. e) Expected Outcome and Complication

1. Union

2. Nonunion

3. Infection

4. Implant failure

\section{f) Data Analysis}

Qualitative variables/Categorical variables were presented in number and percentage (\%), and Quantitative variables/continuous variables were presented as mean \pm SD (whenever required). P-value $\leq 0.05$ was taken as a level of statistical significance. The data was analyzed by SPSS (statistical package for social sciences) Statistical software version 17.0.

\section{Results}

There were five patients (Male -3 , female -2 ). There was no gender difference in the incidence of this fracture. Pediatric age group was found to have a high potential for the union. Long spiral fracture found to be the most common pattern of fracture in our study. There was no failure in our study. The patient's visual analog scale for pain improved in two weeks from an average of 8 to 3 . The radiological first sign of union on x-ray was visible at an average of 4 weeks of fixation. Average Harris hip score was 34 (2wks), 68 (4wks), 87 (6wks), $>90$ (after 2 months). Patients were mobilized with protected weight bearing with some support at 21/2 months. Weight-bearing was gradually increased according to the comfort of the patient. The patients were started long walking at the end of 5 months. The patient started using public transport at the end of 8 months.

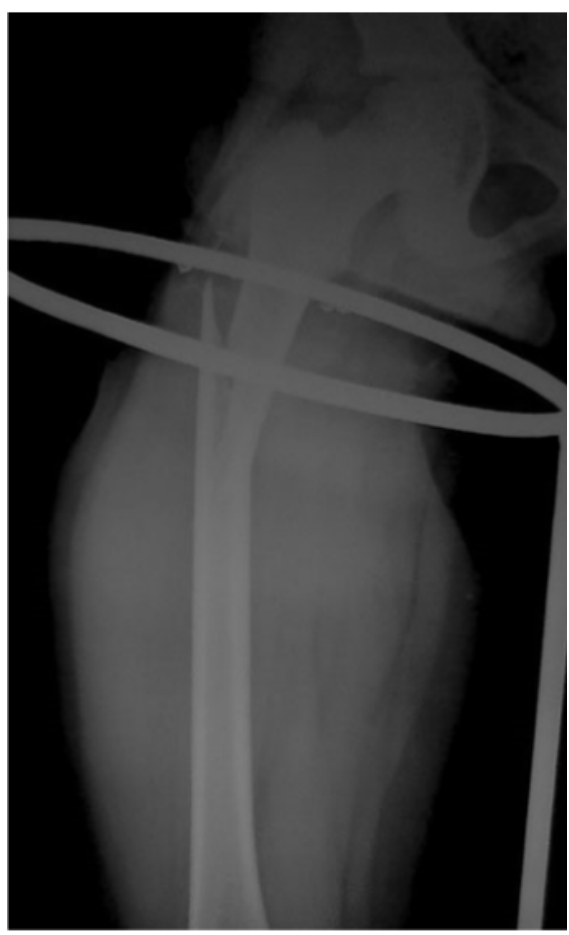

Fig. 1: Preoperative X-ray of the patient 


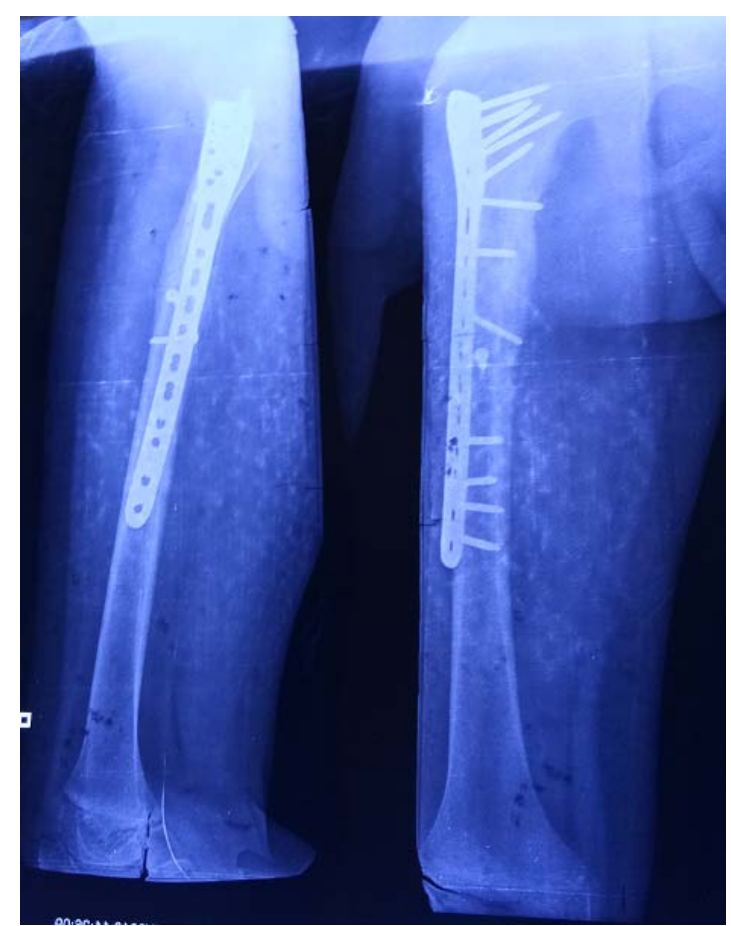

Fig. 2: Postoperative X-ray of the same patient

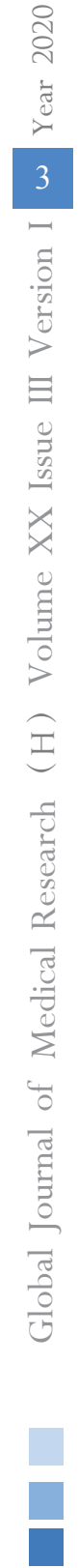

Fig. 3: Final follow up x-ray of the same patient 

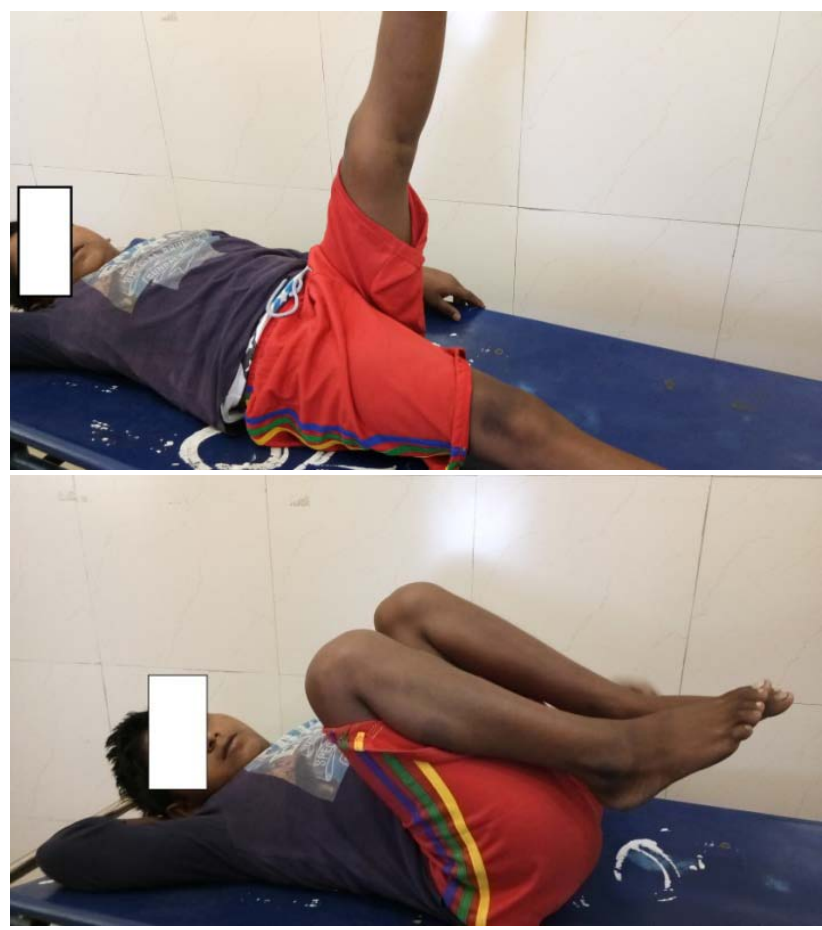

Fig. 4: Function picture of the patient of the same patient

Table 1: Clinical profile of the patient

\begin{tabular}{|c|c|c|c|c|c|c|c|}
\hline $\begin{array}{c}\text { Serial } \\
\text { No. }\end{array}$ & Age/sex & Complication & $\begin{array}{c}\text { Time of } \\
\text { union }\end{array}$ & $\begin{array}{c}\text { Final herris } \\
\text { hip score }\end{array}$ & $\begin{array}{c}\text { Nature of } \\
\text { fracture }\end{array}$ & $\begin{array}{c}\text { End } \\
\text { point }\end{array}$ & Follow up \\
\hline 1 & 11 & - & 12 weeks & 92 & Long spiral & Union & $36 w k s$ \\
\hline 2 & 13 & - & 11 weeks & 91 & Comminuted & Union & 36 wks \\
\hline 3 & 11 & - & 10 weeks & 87 & Short oblique & Union & 36 wks \\
\hline 4 & 14 & - & 14 wks & 95 & Long spiral & union & $36 w k s$ \\
\hline 5 & 12 & - & 10 wks & 89 & Long spiral & union & $36 w k s$ \\
\hline
\end{tabular}

\section{Discussion}

Pediatric subtrochanteric fracture is a rare and unstable type of fracture., ${ }^{4,5}$ Closed displaced subtrochanteric fracture require operative intervention.

Sanders and Egol ${ }^{6}$ presented two cases in which adult, pre-contoured, lower extremity periarticular locking plates were utilized for fixation of subtrochanteric femur fractures in pediatric patients. They proposed that a proximal tibial locking plate in an adolescent and distal tibial locking plate in a young child correspond well to the proximal femur and are thus a viable option in their management.

Cortes et $\mathrm{al}^{7}$ managed atrophic non-union of subtrochanteric femur fractures in an 11-year-old boy using an adult proximal humerus locking plate and packing the non-union site with the demineralized bone matrix. They chose PHLP as they found it to be adequately matched to the surface anatomy of the proximal femur. Six months after the surgery for nonunion, radiographs showed complete union with the maintenance of fracture alignment and morphology of proximal femoral epiphysis. The child was completely asymptomatic with a symmetric range of motion of his hips and knees.

In our study, the proximal humeral locking plate found to be the implant of choice for fixation of any pattern of subtrochanteric fracture. Plate's precontouring found to be well-fitting to the proximal femur lateral surface.

\section{LimitaTiOnS}

There were various limitations to our study. The small numbers of cases due to low incidence, affordability of the patient's attendant, and different patterns of fractures were the limitation. The strength was a single institute and a single operating team. Though we recommend study with larger number of follow-up period with a longer period of follow up.

\section{Vil. Conclusion}

Open reduction and internal fixation with proximal humerus locking plate found to be an excellent implant for fixation of subtrochanteric fracture in adolescent age group. Proximal humerus locking plate found to have an optimum amount of profile thickness 
for the adolescent proximal femur. This plate found to be well-fitting to the proximal femur. Proximal humerus locking plate found to be good for any pattern of subtrochanteric fractures. The direction of locking screw in this plate found to have good purchase in the calcar of neck of femur, which absolute stability for fracture union. Another conclusion drawn to our attention was that lateral decubitus position found to be ideal for adequate reduction of proximal fragment deformities as compared to the supine position. It also provided better visibility. Lateral decubitus posture on the operating table assists in the reduction of the fracture via better visibility and gravity assistance. Direction and length of the locking screws didn't damage the proximal femur physis. Most proximal screws were kept short of physis to avoid damage to proximal femur physis.

\section{References Références Referencias}

1. P. Gogna, M. Mohindra, S. Verma et al. Adult proximal humerus locking plate for fixation of paediatric subtrochanteric fractures. Musculoskelet Surg DOI 10.1007/s12306-013-0310-z.

2. Daum R, Jungbluth $\mathrm{KH}$, Metzger E et al. (1969) Subtrochantere und suprakondylare femur frakturenimkindesalter. Behandlung und Ergebnisse-Chirurgische 40:217.

3. Segal LS (2000) Custom 95 degree condylar blade plate for pediatric subtrochanteric femur fractures. Orthopedics 23(2): 103-107.

4. Staheli LT (1991) Fractures of the shaft of the femur. In: Rockwood CA, Wilkins KE, King RE (eds) Fractures in children, 3rd edn. JB Lippincott, Philadelphia, PA, pp 1121-1142.

5. Sink EL, Gralla J, Repine M (2005) Complications of pediatric femur fractures treated with titanium elastic nails: a comparison of fracture types. J PediatrOrthop 25: 577-580.

6. Sanders S, Egol KA (2009) Adult periarticular locking plates for the treatment of pediatric and adolescent subtrochanteric hip fractures. Bull NYU HospJt Dis 67(4): 370-373.

7. Cortes LE, Triana M, Vallejo F, Slongo TF, Streubel PN (2011) Adult proximal humerus locking plate for the treatment of pediatric subtrochanteric femoral nonunion: a case report. J Orthop Trauma 25(7): e63-e67. 